\title{
Bead-based Microfluidic Sediment Analogs: Fabrication and Colloid Transport
}

Yang Guo ${ }^{1}$, Jingwei Huang ${ }^{2}$, Feng Xiao ${ }^{2}$, Xiaolong Yin ${ }^{2}$, Jaehun Chun ${ }^{3}$, Wooyong Um ${ }^{3}$,

Keith B. Neeves ${ }^{1 *}$, and Ning $\mathrm{Wu}^{1 *}$

${ }^{1}$ Department of Chemical and Biological Engineering, Colorado School of Mines,

Golden, CO, USA 80401

${ }^{2}$ Department of Petroleum Engineering, Colorado School of Mines, Golden, CO, USA

80401

${ }^{3}$ Pacific Northwest National Laboratory, 902 Battelle Boulevard, Richland, WA, USA

99352

\section{Supplementary Information}

\section{Experimental}

The two-layer photolithography procedure for making heterogeneous sediment analogs

For the first layer, KMPR1010 was spin coated at $6000 \mathrm{rpm}$ for $40 \mathrm{sec}$, soft baked for 5 min at $100^{\circ} \mathrm{C}$, exposed through a photomask (CAD/Art service, Bandon, Oregon) to UV light (200 W, 365-405 nm exposure) in a Karl Suss MJB-3 Mask Aligner (Sunnyvale, $\mathrm{CA}$ ) at a dose of $10 \mathrm{~mW} / \mathrm{cm}^{2}$, and hard baked for $2 \mathrm{~min}$ at $100^{\circ} \mathrm{C}$. For the second layer, KMPR 1050 was diluted to $40 \mathrm{wt} \%$ solids in cyclopentanone and spun at $3500 \mathrm{rpm}$ for 40 sec. The same soft bake, exposure, and hard bake procedures were used as the first layer. Both layers were then developed in an aqueous developer solution (AZ300MIF). 
2. Supplementa1 Table 1. Hydraulic resistances of different components in the microfluidic device

\begin{tabular}{ll}
\hline Components & Resistance $\left(\times \mathbf{1 0}^{\mathbf{1 2}} \mathbf{N} \cdot \mathbf{s} / \mathbf{m}^{\mathbf{5}}\right)$ \\
\hline tubing (ID: $0.254 \mathrm{~mm}$, length: $70 \mathrm{~cm})$ & 3.81 \\
post-analog channel & 6.42 \\
empty trap $(1000 \mu \mathrm{m})$ & 1.53 \\
pre-analog channel & 14.59 \\
sediment analog $(1000 \mu \mathrm{m})$ & 63.3 \\
\hline
\end{tabular}

\section{Calculation of hydraulic resistances}

The total resistance of the device, $R$, was calculated by

$$
R=\Delta P / Q
$$

The total resistance includes the resistance of tubing $R_{t}$, the pre/post-analog channel $R_{c}$, and the sediment analog $R_{p}$ connected in series. $R_{t}$, and $R_{c}$ were calculated by

$$
\begin{gathered}
R_{t}=\frac{8 \mu L_{t}}{\pi r^{4}} \\
R_{c}=\frac{12 \mu L_{c}}{W_{c} H_{c}^{3}\left(1-0.63 H_{c} / W_{c}\right)}
\end{gathered}
$$

where $L_{t}$ and $r$ are the length and radius of the tubing, respectively. $L_{c}, W_{\mathrm{c}}$, and $H_{c}$ are the length, width, and height of the pre/post-analog channel. By subtracting $R_{t}$ and $R_{c}$ from the total resistance $R$, we can obtain the resistance of the sediment analog $R_{p}$, which is related to the permeability $\kappa$ by

$$
\kappa=\frac{\mu L_{p}}{W_{p} H_{p} R_{p}}
$$

where $L_{p}, W_{p}$, and $H_{p}$ are the length, width, and height of the analog. 


\section{Supplemental Figures}

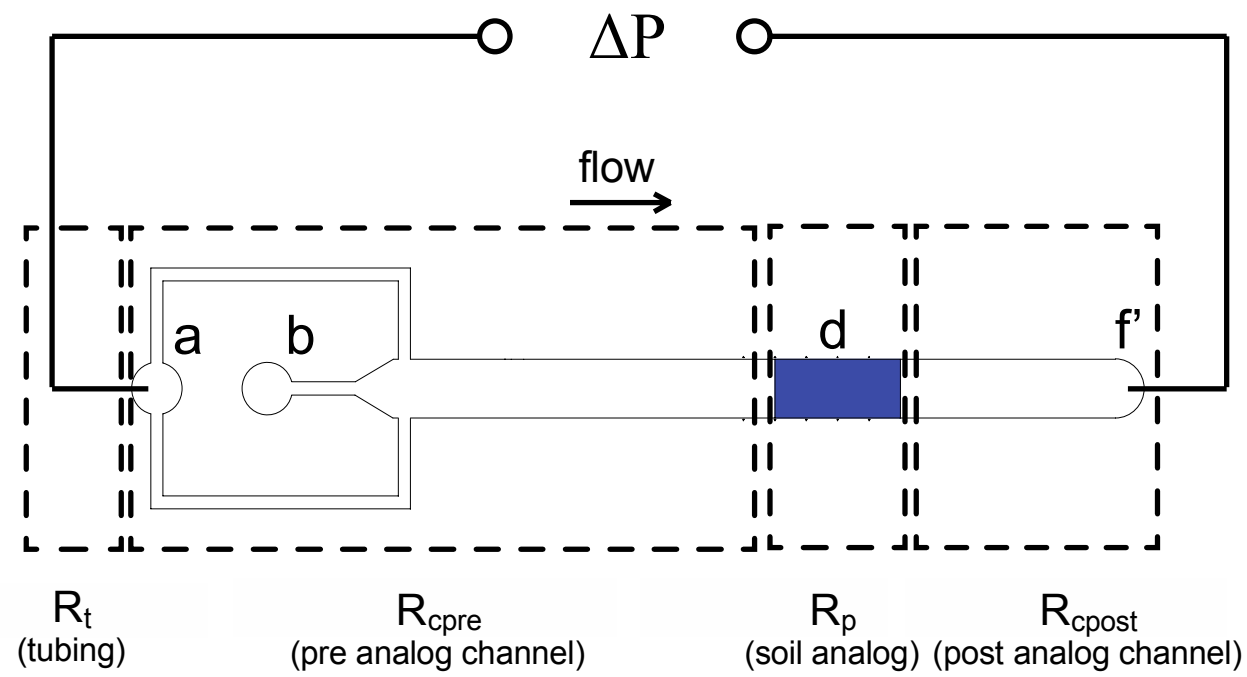

SI Figure 1 The hydraulic resistance circuit for estimating the permeability of the porous medium. 


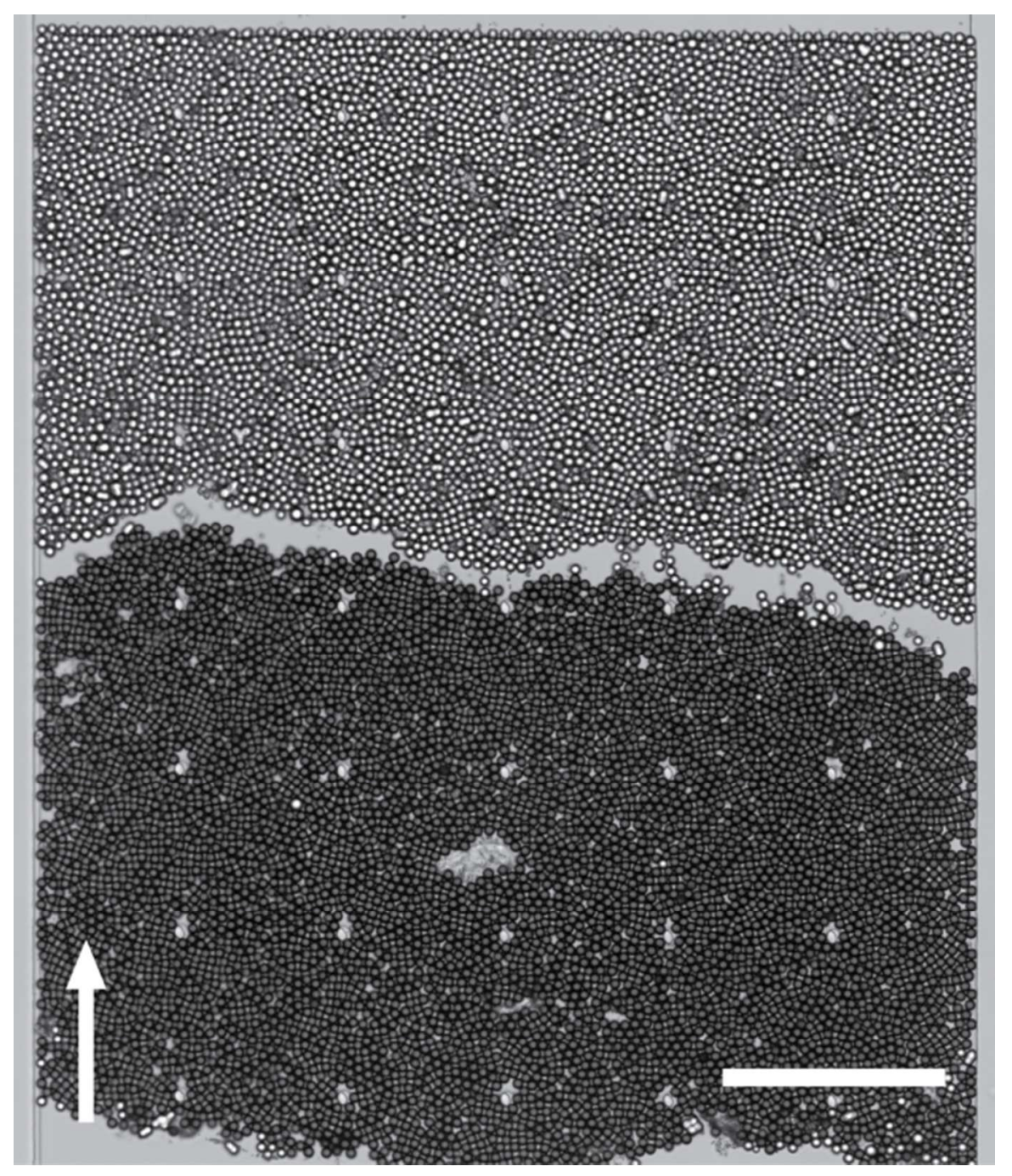

SI Figure 2 The porous sediment analog formed by $10 \mu \mathrm{m}$ aliphatic aminated beads at downstream and $10 \mu \mathrm{m}$ carboxylated beads at upstream. It shows the opposite configuration as in Fig. 2 (B). The arrow indicates the flow direction of colloids. Scale bar: $200 \mu \mathrm{m}$. 


\section{Supplementary videos}

SI Video 1: Bead-filling to form the porous sediment analog. $15 \mu \mathrm{m}$ polystyrene beads were carried by fluid flow from the inlet of the device and they became trapped at the height barrier and then formed the sediment analog by packing along the upstream direction.

SI Video 2: Droplets containing colloids moving through an expansion channel. The dark background in the channel was filled with mineral oil and dye Oil red O. Light green regimes were the aqueous droplets generated at the T-junction. They encapsulated $1 \mu \mathrm{m}$ fluorescent colloids (brighter green dots) that have been flown through the porous medium.

SI Video 3: Individual colloid trajectories. $1.8 \times 10^{-4} \mathrm{wt} \%$ of $1 \mu \mathrm{m}$ fluorescent PS colloids solution was used in the experiment. The exposure time was $500 \mathrm{~ms}$ to capture the trajectories of individual colloids.

SI Video 4: Colloids re-mobilization and re-adsorption on the positively charged beads section placed downstream during flushing. 